\title{
1 Visual Response Properties in the Three Layer Turtle
}

\section{Visual Cortex}

4 Mahmood S. Hoseini ${ }^{* 1}$, Jeff Pobst ${ }^{1}$, Nathaniel C. Wright ${ }^{1}$, Wesley Clawson ${ }^{2}$, Woodrow

5 Shew $^{3}$, Ralf Wessel ${ }^{1}$

6

$7 \quad{ }^{1}$ Department of Physics, Washington University, St. Louis, MO

$8 \quad{ }^{2}$ Department of Electrical Engineering and ${ }^{3}$ Department of Physics, University of

9 Arkansas, Fayetteville, AR

10

11 Contact information: Corresponding author email - sayedmahmood@wustl.edu 


\section{ABSTRACT}

13 Turtle dorsal cortex provides us with unique insights into cortical processing. It is

14 known to share many features with the mammalian hippocampus and olfactory cortex

15 as well as geniculo-cortical areas in stem amniotes from which mammals evolved. To

16 this end, we have used data from extracellular recordings from microelectrode arrays to

17 study spatial and temporal patterns of responses to visual stimuli as seen in both local

18 field potential and action potentials. We discovered surprisingly large receptive fields,

19 responsiveness to a broad range of stimuli, and high correlation between distant neural

20 ensembles across recording array. Moreover, we found significant response variability

21 regarding latency and strength in the presence of adaptation to both ongoing and 22 visually evoked activity.

24 Key Words: (4-5) Visual Cortex, Receptive Field, Receptive Field, Response

25 Spatiotemporal Properties 


\section{Introduction}

27 Understanding evolutionary origins of the mammalian cortico-thalamic system will infiltrate our knowledge. The study was launched through reptilian brains since they are simpler that their mammalian counterparts (Butler and Hodos, 2005; Naumann et al., 2015). Comparing cortical circuits between mammals and reptiles opens up a window into the role of different brain areas in cognitive processing (Naumann et al., 2015). Turtles are of particular interest for comparative studies because they probably bear the strongest resemblance to the Triassic cotylosaurs (stem amniotes) from which they and all modern mammals evolved (Romer and Parsons, 1977). Concomitant with comparative studies characterizing spatiotemporal features of visual responses helps us to uncover the underlying circuitry in mammalian cortex and, ultimately, understanding

37 how the cortex processes sensory information.

The turtle dorsal cortex is a convergent zone for the visual, auditory, somatic, and other sensory systems (Gusel'nikov et al., 1972). Nonetheless, it has mostly been studied with respect to visual processing. There are several studies on the spiking response to diffuse flashes (Gusel'nikov et al., 1972; Kriegstein, 1987; Mancilla et al., 1998) and moving black dots (Gusel'nikov and Pivovarov, 1977) as well as the size and organization of the receptive field (RF) of spiking cells (Mazurskaya, 1973a). However, there have only been very few studies focusing on voltage sensitive dyes (Prechtl et al.,

45 1997) or local field potential (LFP; Bass et al., 1983; Prechtl, 1994; Prechtl and Bullock, 46 1994; Prechtl et al., 2000; Luo et al., 2010). Nevertheless, there exist very little 47 agreement about different features of response such as RF size, adaptation, and 
direction tuning (Gusel'nikov et al., 1972; Mazurskaya, 1973a; Boiko, 1980; Mulligan

49 and Ulinski, 1990; Luo et al., 2010).

Here, we have revisited some of these questions. The clues at our disposal to

51 explore response spatiotemporal properties are extracellular recordings from

52 microelectrode arrays (MEA) that capture key integrative synaptic processes in neural

53 networks. We have found large RFs that typically cover over half of the visual field with

54 no indication of direction selectivity in line with previous study (Boiko, 1980).

55 Investigating spatial structure of the receptive fields, we have discovered no clear or

56 well-defined retinotopic map from the visual field (VF) to the cortex. Furthermore, our

57 results demonstrate a broad range of response latencies and a strong adaptation to

58 both ongoing and visually evoked activity.

\section{Materials and Methods}

\section{Ex Vivo Eye-Attached Whole Brain Preparation}

All procedures were approved by Washington University's Animal Care and Use

63 Committees and conform to the guidelines of the National Institutes of Health on the

64 Care and Use of Laboratory Animals. Adult red-eared turtles (Trachemys scripta

65 elegans, $150-200 \mathrm{~g}$ weight, $12-15 \mathrm{~cm}$ carapace length) were studied. Rapid

66 decapitation was performed following anesthetization with Propofol $(10 \mathrm{mg} / \mathrm{kg})$ (Ziolo

67 and Bertelsen, 2009). We then removed the brain with the right eye attached and 68 proceeded to hemisect the eye. 
To access the ventricular surface of the left visual cortex, we cut off $\sim 1 \mathrm{~mm}$ of the left olfactory bulb, which provided a hole to start a rostral-caudal cut through the medial cortex. This cut continued from the olfactory bulb into the natural separation of the

72 medial cortex from the septum (about $1 / 3$ of the cortex) and continued further along the same line for $\sim 1-2 \mathrm{~mm}$ into the caudal cortex. Finally, two cuts were made from the medial cortex edge towards the dorsal cortex. These two cuts were started at roughly $1 / 3$ and $2 / 3$ the rostral-caudal length of the cortex and were made as short as possible $77 \sim 2 \mathrm{~mm}$.

After making the cuts in the cortex, it was placed in the recording chamber on either a Sylgard or agar surface, and insect pins were used to pin the cortex flat. Our electrodes were then placed in the flattened cortex. The eye and brain were continuously perfused with artificial cerebrospinal fluid (in mM; $85 \mathrm{NaCl}, 2 \mathrm{KCl}, 2 \mathrm{MgCl}_{2}$, $45 \mathrm{NaHCO}_{3}, 20 \mathrm{D}$ glucose, and $3 \mathrm{CaCl}_{2}$ bubbled with $95 \% \mathrm{O}_{2}$ and $5 \% \mathrm{CO}_{2}$ ), adjusted to $\mathrm{pH} 7.4$ at room temperature. To perfuse the eye without obstructing the image we project onto the retina, a small wick was made from a Kimwipe. The wick connected an artificial cerebrospinal fluid (ACSF) feed located $\sim 1 \mathrm{~cm}$ above and to the side of the eye to the inside edge of the hemisected eye. If any brain tissue were large enough to

87 extend above the surface of the ACSF (e.g., the right cortex or the optic tecta), it would be cover with a small piece of Kimwipe so that it would also stay in contact with ACSF. Recordings began 2-3 hours after anesthetization. 
91

92

\section{Visual Stimulation}

For the included studies, three methods of visual stimulation were used.

LED Stimulation. For LED stimulation, a red LED was connected to the output of a National Instruments BNC-2090 terminal block connected to a National Instruments PCI-6024E DAQ board. This output was controlled with a custom LabView program on a computer running Windows 7 . The mean light intensity at the retina was $60 \mathrm{~mW} / \mathrm{m}^{2}$.

Monitor/Mirror Stimulation. For monitor/mirror stimulation, a 19" LCD monitor (Samsung model Syncmaster T190, 1440x900 pixels, contrast ratio=20000:1, response time $=2 \mathrm{~ms}$ ) displayed the stimuli. This image was reflected off a mirror located across room above the tissue, and focused on the retina with a lens placed above the tissue (Fig. 1a). The mean light intensity at the retina from the monitor was $20 \mathrm{~mW} / \mathrm{m}^{2}$.

Projector Stimulation. For projector stimulation, a small projector was placed above the retina and focused with a system of lenses (Aaxa Technologies, P4X Pico Projector, $1440 \times 900$ pixels). The mean light intensity at the retina from the projector was $1 \mathrm{~mW} /$ $m^{2}$. Both monitor/mirror and projector stimulation was provided using software written in python on a computer running Ubuntu 10.4. Visual stimuli included black dots moving on a white screen, naturalistic video, and red LED flashes.

\section{Data Acquisition}


Microelectrode Array Recordings. Data were collected at a $30 \mathrm{kHz}$ sampling rate using the Cerebus data acquisition system by Blackrock Microsystems. Two different

112 styles of microelectrode arrays were used for our recordings. For some recordings, we used a 96-channel Utah array (Fig. 1b; 10x10 square grid, $400 \mu m$ inter-electrode

114 spacing, $500 \mu m$ electrode length, no corner electrodes, Blackrock Microsystems). For 115 others, we used an array of shank electrodes (4x4 array of shank electrodes with 8 116 recording sites on each electrode, $300 \mu \mathrm{m}$ and $400 \mu \mathrm{m} \times$ and $\mathrm{y}$ distance between 117 shanks and $100 \mu \mathrm{m}$ between recording sites along a shank, Neuronexus). We attached 118 either array to a post fastened to a micromanipulator (Sutter, MP-285). The Utah array 119 was inserted to a depth of $250-500 \mu \mathrm{m}$ starting from the ventricular side of the 120 unfolded cortex such that the plane of electrodes was parallel to the dorsal surface of 121 the cortex. The array of shank electrodes was inserted deep enough to span the entire 122 depth of the cortex.

We recorded wideband $(0.7 \mathrm{~Hz}-15 \mathrm{kHz})$ extracellular voltages relative to a silver 124 chloride pellet electrode in the tissue bath.

126 electrodes, we used tungsten electrodes (500k $\Omega$ part \#WE30030.5H5 from

127 MicroProbes and $1000 \mathrm{k} \Omega$ catalog \#573520 from AM Systems). For some experiments, 128 we also used homemade tetrodes with resistances between $250 \mathrm{k} \Omega$ and $350 \mathrm{k} \Omega$. These 129 were made by twisting four $12.7 \mu \mathrm{m}$ nickel chromium wires together, applying heat with 130 a heat gun (Weller 6966C) and cutting the twisted wires at an angle to expose the ends 
131 for recording (Saha et al., 2013). Recordings were taken in reference to a silver chloride 132 ground wire sitting in the bath.

The signals from these electrodes were recorded with an AM Systems Model 1800 amplifier connected to a National Instruments PCI-6024E 12-bit DAQ board through a National Instruments BNC-2090. The data were collected at $20 \mathrm{kHz}$ using Labview.

\section{Filtering for Local Field Potential}

To study LFP, it is useful to filter out other frequencies. For our LFP analysis, we used the PyWavelets package to perform wavelet filtering (Wiltschko et al., 2008). We used Daubechies wavelets with a minimum level of 9 and a maximum level of 11 . For our $30 \mathrm{kHz}$ data, this corresponds to a pass-band of $\sim 7-59 \mathrm{~Hz}$.

Defining an LFP Event. There are many ways one can quantify the size of an LFP response. One method we use throughout this paper is to look for threshold crossings

144 of the extracellular signal after filtering it to the frequencies we are interested in. As a 145 threshold for LFP events we used 3 times standard deviation of the filtered signal (Fig. 146 1c). Therefore, when we refer to LFP event count, we are simply referring to a number 147 of threshold crossings. electrodes of the MEA, only a subset of electrodes would actually have a strong visual 
150 response. In order to get clean results, it is necessary to do our analysis on only that 151 subset of electrodes.

To consistently and systematically determine which electrodes to include, we created an algorithm to test for visual responsiveness. Roughly speaking, an electrode was considered visually responsive if the typical level of activity following visual 155 stimulation was sufficiently greater that spontaneous ongoing level activity. Specifically, we established the spontaneous ongoing level of activity, $a_{\text {ong }}$, by taking the average

157 activity from $4 s$ windows immediately preceding the presentation of the stimulus. 158 Similarly, the amount of visually evoked activity, $a_{\text {evoked }}$, was the average of the $4 s$ 159 windows immediately following the onset of the visual stimulus. We then calculate the 160 decrease in activity (how much lower is the ongoing activity level than the evoked 161 activity level) as $D=1-\frac{a_{\text {ong }}}{a_{\text {evoked }}}$. Finally, we classify an electrode as visually responsive 162 if the decrease in activity is greater than 0.75 (Fig. 1b).

\section{Defining Receptive Field Similarity}

To quantify RF similarity between nearby electrodes, we calculated the amount of

167 paths in visual field (bin size $=8$ visual degrees), calculated the normalized average LFP response to stimulation in each of those bins, and for each electrode pair, we normalized response values (one for each electrode) over all bins. 
The normalization of the average LFP response in each bin was done by dividing

172 the average response by the sum of the average responses over all bins (or, in the case

173 of direction specific RF similarity, by dividing by the sum of the average responses over

174 only the bins for the angle of interest). Consequently, the sum of the normalized

175 responses over all bins was always one, the minimum similarity between two electrodes

176 was zero, and the maximum was one.

178 RESULTS

\section{Overview}

To quantify the spatiotemporal structure of visual responses we recorded

extracellular neural activity using MEAs inserted into the geniculo-recipient dorsal cortex

182 of the turtle eye-attached whole-brain ex vivo preparation during visual stimulation of the

183 retina (Fig. 1a). First, we explore spatial properties in terms of RF size, RF similarity, 184 and direction sensitivity; and then temporal features are quantified in terms of response 185 duration, latency, and adaptation.

\section{Spatial Properties}

LFP traces were recorded in response to black dots moving across a white

189 background (8 degrees diameter dot moving at $40 \mathrm{deg} / \mathrm{s}$ ). Dots move in several different directions (either 4 or 8 different angles), and for each angle, dots move along 
1918 different straight paths spanning the visual field. Our probe of the spatial structure 192 includes RF size, RF similarity, and direction selectivity and is as follows.

\section{1. RF size}

LFP in response to moving dots on the screen is used to determine the size of RF.

195 For each moving dot along a path, we mark LFP events of a given electrode (Fig. 1c; see Materials and Methods). In order to aid visualization, LFP events are plotted

197 somewhat offset from the actual path of the presented dots. Events are color coded 198 according to the direction of the moving dots. Responses in each direction over different trials and paths are summarized in the distribution of the average LFP event count with the trial-to-trial variability shown as the filled region around the average line (Fig. 2).

Typically, near the edges (for the first and last paths) LFP response diminishes.

202 This is due to the fact that for those paths dots spend less time moving in the visual field 203 (Fig. 2). More importantly, the RF of the LFP seems to span large areas of the visual 204 field in different preparations (Fig. 3) as well as across different electrodes of MEA (Fig. 205 4). We see that RFs commonly cover over half of the visual field.

Looking at the RFs plotted across MEA, it appears that the RFs of nearby electrodes are more similar to each other than those of distant electrodes (Fig. 4 and

209 Fig. 5a). To quantify the similarity of RFs between pairs of electrodes, we calculated the 210 amount of overlap by binning the dots paths in the visual field to calculate the 211 normalized average LFPs. Then we sum the smaller of the two normalized response 
212 values over all bins and divide it by the sum of the average responses over all bins (see

213 Materials and Methods). To determine the significance of similarity between two

214 electrodes, we recalculated the similarity between the two electrodes after shuffling the

215 binned responses of one of the electrodes. This process was done 1,000 times. We

216 then call the original similarity significant if it is higher than $95 \%$ of the shuffled

217 similarities.

Our analysis demonstrates that neighboring electrodes have significantly similar

219 RFs, consistent with our qualitative conclusion (Fig. 5b). Regardless of the direction of

220 moving dots, RFs of the given pair exhibit a significant similarity of 0.46 . Now the

221 question remains to be answered is that does RF similarity depend on the spatial

222 separation between electrodes? For the four turtles that had several visually responsive

223 electrodes, we plotted the similarity versus distance for a single electrode paired with all

224 other electrodes. Then we made this plot for all visually responsive electrodes, and

225 finally, we arranged these plots in the same way the corresponding electrodes are

226 arranged on the MEA (Fig. 6a). Mostly negative slopes indicate that RFs of nearby

227 electrode pairs tend to be more similar to each other than the RFs of distant electrode

228 pairs. The average RF similarity at each electrode pair distance for all visually

229 responsive electrode pairs for four turtles indicates a consistent decay (Fig. 6b).

230 Additionally, it appears that the negative slope can be found more consistently for 231 the rostral electrodes than for the caudal electrodes (Fig. 6a). The caudal electrodes

232 tend to have slopes closer to zero. This means that the RFs at caudal electrode sites 233 are no more (or only slightly more) similar to their neighbors than they are to distant 
234 electrodes. This is seen more clearly when looking at a larger section of the array and is

235 a consistent result across turtles (data not shown). It is worth noting that in a majority of

236 cases; even the lower levels of similarity are still significantly more similar than shuffled

237 data.

238

239

240

241

242

243

244

245

246 line distributions in Figs. 2, 3, and 5) further support this hypothesis. Overwhelmingly,

247 we discovered that the average response curves to opposite directions are nearly mirror

248 images of each other (see Fig. 2 for example). This indicates that there is no opposite 249 angle direction sensitivity in the LFP response (when quantifying the LFP response as 250 the number of threshold crossing of the LFP).

\section{Temporal Properties}

So far we have looked at the spatial structure of the responses. One equally

254 important topic to explore is the time course of the responses. How long do responses 
255 to brief and extended stimuli last? How long is the response latency in the visual cortex?

256 How does the strength of responses adapt? Does adaptation depend on stimulus 257 characteristics?

LFP signals in response to brief LED flashes $(50 \mathrm{~ms})$ often contain oscillations that

259 last for several hundreds of millisecond regardless of the flash amplitude (Fig. 7a).

260 While at earlier times after flash onset these oscillations are clearly dominated by one or

261 two frequencies, at later times, they are made up of fluctuations covering a broad range

262 of frequencies (Fig. 7a). Rather than characterizing power spectrum of the responses,

263 we quantify the temporal properties of LFP events after flash onset in terms of response

264 duration, latency, and adaptation.

265

266

267

268

269

270

271

272

273

274 275 activity up to $10 \mathrm{~s}$ after the presentation of a flash (Fig.8).

\section{Response duration}

For each trial and flash amplitude, we determine threshold crossings after stimulus onset, and then sum over trials to obtain prestimulus time histogram (PSTH; Fig. 7b, c, and d). We typically see responses that last $500 \mathrm{~ms}$ to $1 \mathrm{~s}$ depending on the flash amplitude. In addition, when looking at visually evoked LFP activity over an extended period of time, in many instances, we also see a second (or even third) period of activity after periods of relative inactivity even several seconds after flashes (Fig. 8). This persistent activity occurs on different electrodes of all preparations (Fig. 8) even in response to very brief flashes $(10 \mathrm{~ms})$. If we compute PSTHs for several electrodes and arrange them based on their location in MEA, we see a reliably reproducible wave of

\section{Response latency}


Our results involve relating the activity recorded in the cortex with the stimulus presented to the retina. One important aspect of this relationship is how much delay

279 there is between the presentation of the stimulus and the response caused by signal 280 propagation time in the pathway leading to the cortical response. This is an interesting 281 question per se, but also an essential piece of information when it comes to interpreting 282 the responses to stimuli that cannot be characterized as occurring at only one instance 283 in time.

We investigated the latency between stimulus presentation and the first evoked 285 spike recorded on extracellular electrodes. To this end, we looked at the latency to the first spike in response to stimuli with a precise ON time covering the entire visual field.

287 When looking at responses to a full-screen flash or to the change from a blank screen to 288 the start of a complex movie, we found an extensively wide range of latencies (Fig. 9). 289 Our results indicate that a typical latency to first evoked spike is around $200-500 \mathrm{~ms}$ 290 (Fig. 9). A further support for this delay appears through RF analysis. For each LFP 291 event detected, if we mark the region of the visual field in which the dot was at earlier 292 times $(0,150,300 \mathrm{~ms})$, we see the most overlap of the contributions to the visual field 293 from the dots moving at different angles (Fig. 10). As such, for all figures showing the 294 RF as probed by moving dots, a $300 \mathrm{~ms}$ delay has been applied.

\section{Adaptation}

296 The effects of adaptation in turtle visual cortex are clear, long lasting, and 297 ubiquitous. An adapted response in the visual cortex can be the result of two different 298 sources: The cortex may be adapting in such a way that it has a diminished response 
299 (relative to the unadapted state) to the same cortical input, or adaptation has taken place at an earlier stage in the visual pathway, and the cortex is still responding in a consistent way as before adaptation but to a diminished cortical input. To determine whether cortex has an adaptation mechanism, we looked at responses in the presence or absence of prior stimulus.

Visual-Visual Adaptation: To test the effect of a stimulus on a subsequently presented stimulus, we used moving dots, radially moving bars, and full field flashes. In the clearest demonstration of adaptation, when we presented a series of $100 \mathrm{~ms}$ duration LED flashes to the retina, we reliably recorded a strong LFP response to the first flash, and either no response or a greatly diminished response to the subsequent flashes presented $2 s$ afterward (Fig. 11a). The extent to which the subsequent responses were diminished depended on the time separation of the flashes. Consistent with an earlier study (Luo et al., 2010), this dependence was not all-or-none; in between the short inter-flash-intervals that completely abolished subsequent responses and the long interflash-intervals that seemed not to affect subsequent responses, there were intermediate inter-flash-intervals that resulted in somewhat diminished subsequent responses.

We further demonstrated the effects of adaptation with more complex stimuli containing spatial and temporal structure. To quantify response adaptation to

317 subsequent stimuli, we used dots moving along different paths through the visual field.

318 For 8 different angles, we moved dots across 5 paths in the visual field in an ordered 319 sequence. For opposite angles, the paths overlapped but were in reversed order, e.g. 320 the path that was presented first at an angle of 0 degrees was presented last at 180 321 degrees. This allowed us to compare the response to presentation order while 
322 controlling for the area of the visual field being stimulated.

323

To quantify the effects of adaptation due to stimulus presentation order, we defined

324 the decrease in response, $d$, as simply the average of the decreases for individual paths

325 according to $1-\frac{r_{\text {late }}}{r_{\text {early }}}$, where $r_{\text {late }}$ is the strength of the response when the path was

326 presented late in the series (either the fifth or fourth path to be presented), and $r_{\text {early }}$ is

327 the strength of the response when the path was presented early in the series (either first

328 or second).

When we look at the response amplitude when a path was presented first compared

330 to the same path being presented fifth (or second compared to fourth), we clearly see

331 adaptation of responses to stimulation of one area of the visual field caused by previous

332 stimulation of other areas of the visual field (Fig. 11b).

On average, the evoked response to the fifth path is $76 \%$ smaller than when it is the

334 first presented path. Similarly, fourth path response is $82 \%$ smaller than the second

335 path response. The fact that the decrease in response strength is larger and more

336 reliable for the second-fourth pairs than it is for the first-fifth pairs is most likely due to

337 complications near the edge of the visual field. The first/fifth paths are always on the

338 very edge of the visual field. Consequently, the dots moving along those paths were not 
341 interior paths. As such, these weaker responses may be more confounded by noise.

342

343

344

345

346

347

348

349

350

351

352

353

354

355

356

357

358

359

360

361

362

In a different set of experiments, adaptation to visual stimuli was studied while controlling for not only same path in the visual field, but also the direction of motion along that path. In contrast to the previous data set, in this data set the order in which the paths were traversed was randomized for each trial. Thus, a given path may have been the first path presented during one trial, but the fourth path presented during the next. This allowed us to separate the responses to a dot moving along any given path into trials for which the path was the first path to be presented and trials for which the path was not the first path presented. Using the same LFP threshold crossing described earlier, for each path, we calculated two average responses: the average first-presented response and the average non first-presented response. For this data set, time interval between dots moving in a given direction is $10 \mathrm{~s}$. After presenting all 5 paths, there is either $118 s$ or $214 s$ waiting time before starting a new set of 5 dots in a different direction. In Fig. 11c the average response when a path was first is plotted against the average response to that same path when the path was not first. Because there were very few trials for any given angle-path combination, the results are somewhat scattered. But, when taken as a whole, for the 575 responsive points shown, average non first-path responses are significantly weaker than the average first-path responses ( $p=1.7 E-20)$.

Ongoing-Visual Adaptation: While the results so far clearly demonstrate an adapted response to visual stimuli, they do not shed any light on the sources of adaptation. It is unclear if the adaptation is taking place in the cortex, at an earlier stage in the visual 
363 pathway, or (most likely) some combination of both effects. To better understand

364 adaptation happening within the cortex, we looked at how visual responses adapted to

365 spontaneous activity within the cortex (Fig. 11d). Here we looked at repeated trials of a

366 given stimulus and picked out the trials that had a large burst of LFP events within the

$3675 s$ leading up to the stimulus presentation. We then calculated the average response to

3684 trials of the same stimuli that did not have a large spontaneous burst preceding them.

369 To avoid having our results confounded by experimental rundown, we selected the 4

370 trials that occurred most closely in time to the trial that was preceded by spontaneous

371 activity. It is clear that spontaneous activity in the cortex can lead to a significant and

372 reliable adaptation of subsequent visual responses (Fig. 11d). Although our results do

373 not disentangle adaptation in the cortex from adaptation in pathways leading to the

374 cortex, it indicates that the cortex has an adaptation mechanism by itself such that

375 ongoing burst of activity diminishes subsequent response strength.

377 Response Variability

378 Despite response characterization so far, we see large a variability in the responses

379 to repeated presentations of the same stimulus. This variability manifests itself in 380 different ways from variable strength to variable timing or even variable temporal and 381 spectral properties (OUR PAPER).

Looking at the responses to moving dots, there seem to be two different visual responses to dots moving along the $3^{\text {rd }}$ and $4^{\text {th }}$ paths (Fig. 12). If we focus on the 384 responses to the $3^{\text {rd }}$ path, we see that of the 16 trials, there are only responses in 5 or 6 
385 386

of them (first of those "responses" is likely a spontaneous activity, since it starts slightly before the stimulus). On the other trials, there are no visible LFP oscillations. Two of the non-responding trials might have been affected by adaptation from the bursts of activity preceding the stimulus but that still leaves 8 non-responsive trials. Similarly, the nonresponsive trials for the $4^{\text {th }}$ path are likely due to the responses to the $3^{\text {rd }}$ path that occurred just before the $4^{\text {th }}$ path. Interestingly, this all-or-none response variability was not seen for dots moving in the opposite direction along the same paths in a set of recordings taken over the same period of time (data not shown).

We also see variability in the temporal structure of the response (Fig. 12). Not only do responses to both the $3^{\text {rd }}$ and the $4^{\text {th }}$ path have substantial differences in the time to response onset (sometimes varying by as much as a second), but they also vary in how that response plays out. For some trials (e.g., trials 1-3) we see roughly one large oscillation, and for others (e.g., trial 4) it looks more like a series of two smaller bursts.

Finally, Fig.12 also contains examples of response strength variability. If we compare the responses to the $3^{\text {rd }}$ path in the $7^{\text {th }}$ and $8^{\text {th }}$ trial, we find markedly different amplitudes of response. These interesting observations worth exploring further in future studies.

\section{Spike-LFP Correlation}

In general, spikes are much less common in the absence of LFP activity than they are during a burst of LFP activity and there is a clear positive correlation between the number of action potential and the number of LFP peaks (Shew et al., 2015). Here we 
407 look at the RF size using detected spikes and then compute its similarity to the RF 408 obtained using LFP events. Again, spike RFs cover a large region of the visual field 409 and, in other words, units fire action potentials in response to dots presented at different 410 locations of the visual field (Fig. 13a). However, spikes have a less reliable trial-to-trial 411 variability since they are less common than LFP events. More importantly, spike RF is 412 exceptionally ( $80 \%$ ) similar to the RF obtained using detected LFP events on the same 413 electrode (Fig. 13b; average overall similarity $45 \pm 15 \%$, mean $\pm s t d$ ).

\section{Possible Mapping of the Visual Field to the Visual Cortex}

For decades it has been reported that pyramids in turtle visual cortex respond to small moving stimuli spanning a very large portion of the visual field. While our results

417 show large RFs for both LFP events and spiking activity, when we looked for more 418 detail in the spatial structure of the RFs, there is less of a consensus regarding what to expect. Though there is not a clear and well-defined retinotopic map to the cortex, there have been a few studies that report on projection at two steps leading from the retina to

421 the cortex. It has been hypothesized that naso-temporal axis of visual space is 422 represented along the rostro-caudal axis of the visual field (Mulligan and Ulinski, 1990).

423 However, this prediction contradicts earlier results indicating opposite polarity in 424 recorded evoked potentials in response to localized visual stimulation of the retina 425 (Mazurskaya, 1973a).

To resolve this puzzle, we used moving dot stimuli and investigated whether the 427 nasal-temporal response specificity changes as we compare data from electrodes in the 428 rostral cortex with those from caudal cortex. By performing the same experiments with 429 the visual stimuli rotated 90 degrees, we were able to investigate dorso-ventral 
430 response specificity. We found that neither Mazurskaya's results nor Mulligan and

431 Ulinski's predictions are consistent with our findings.

432 Naso-temporal Visual Field. We tested this with moving dots that followed straight paths

433 from the top of the visual field to the bottom (as well as dots moving in the opposite

434 direction). Eight of these vertical paths were spread out at different naso-temporal

435 locations spanning the visual field. Only one dot (following one path) would move at a

436 time. After moving these dots along the different paths we could look for naso-temporal

437 response specificity at any given recording site in the cortex to see if the nasal-temporal

438 response specificity changes as we compare data from electrodes in the rostral cortex

439 with those from the caudal cortex.

440 Our results show that, as you compare the naso-temporal response specificity of 441 different recording sites, the strength of response specificity does change, but the 442 pattern of specificity was approximately the same for all recording sites, and the 443 variations from site to site did not follow any clear trend (e.g., rostral recording sites 444 responding strongly to one area while caudal sites respond strongly to a different area; 445 Fig. 14a).

Dorso-Ventral Visual Field. By performing the same experiments with the visual stimuli

447 rotated 90 degrees, we investigated response specificity for different elevations in the 448 visual field. In contrast with previous predictions, we found recording sites that 449 responded clearly to only the upper or only the lower visual field rather than stimulation 450 at all elevations (Fig. 14b). Overall these findings strongly support no well-defined 451 mapping from the visual field to the visual cortex. 


\section{DISCUSSION}

Here, we investigated the basic spatiotemporal properties using MEA recordings of 455 visual responses in turtle whole-brain eye-attached ex-vivo preparations while 456 stimulating retina primarily with black moving dots in different directions. We found large 457 RFs that typically cover over half of the visual field with no indication of direction 458 sensitivity. Our investigations revealed no clear or well-defined retinotopic map from the 459 visual field to the cortex. Additionally, a broad range of response latencies and a strong 460 adaptation to both ongoing and visually evoked activity have been observed.

We know that the size of RFs in lateral geniculate nucleus (LGN) cells is restricted to 30 degrees (Boiko, 1980) and that there are thalamic axons connecting to the cortex with RFs as small as 2-5 degrees (Mazurskaya, 1973a). Then how does large cortical 464 RF settle with small RF found in the LGN? Two explanations are conceivable here. It could be that an individual cortical cell has a large RF because it receives input from many LGN cells whose RFs collectively span a large area of the visual field, but these seems unlikely given the proposed projections from LGN to cortex (Mulligan and Ulinski, 1990). Therefore, what seems more likely is that the large cortical RFs may be the result of individual cortical cells receiving input from many other cortical cells that each

470 receives LGN input representing only a small portion of the visual field.

While it is important to understand the thalamic inputs to the cortex, we firmly

472 believe that intracortical connections play an essential role in determining the RF size 473 and structure. The extent to which the RF of individual cells in the visual cortex depends 
474 on the connectivity with other cortical cells has been demonstrated by comparing the

475 normal RF of a cortical cell to its RF after applying pharmacological blockers to different

476 areas of the cortex (Mazurskaya, 1973b). In support of our viewpoint, this study found

477 that after applying blockers to other areas of the cortex, there would be gaps in the large

478 RF that previously were not present. This suggests that for that cell, its responsiveness

479 to certain regions of the visual field depended on receiving signals from the blocked 480 region of the cortex.

How the RF similarity of nearby pairs can be interpreted? It might suggest that LFP 482 reach is larger than the inter-electrode distance $(\sim 400 \mu \mathrm{m})$ so that neighboring 483 electrodes are essentially measuring the same signal. While there have been some 484 recent claims that the spatial extent of the LFP can be as large as several millimeters 485 (Kajikawa and Schroeder, 2011), it has usually been thought that the LFP represents 486 neural activity within roughly $150-400 \mu \mathrm{m}$ of the electrode (Katzner et al., 2009; Xing 487 et al., 2009). The fact that we occasionally see bursts in narrow frequency bands on one 488 electrode but not on the adjacent electrode (data not shown) is consistent with a smaller 489 spatial extent for the LFP. This suggests that the similar RFs recorded at different 490 electrodes (spaced $400 \mu \mathrm{m}$ apart) are not merely measurements of the same signal 491 generated by common sources, but are instead measurements of activity generated by 492 different sources. A reasonable speculation is that they happen to produce similar 493 signals due to immense recurrent connections among neurons.

Our findings overwhelmingly point to no direction sensitivity in moving dot 
496 only $9 \%$ of units in turtle thalamus show direction sensitivity, though the significance of 497 these results is questionable (Boiko, 1980). Whether or not direction sensitivity exists in 498 the thalamus affects how we think about its occurrence in the cortex. In either case, we 499 have to take synaptic connection structure into account to explain direction insensitivity 500 of cortical neurons to given thalamic inputs.

It takes time for the signal elicited by visual stimuli to travel from the retina to the 502 thalamus and then to the cortex. Many studies have reported first spike latencies in 503 visual cortex to be between $80 \mathrm{~ms}$ and $200 \mathrm{~ms}$ (Mazurskaya, 1973a) and even as short 504 as $25-150 \mathrm{~ms}$ (Bass et al., 1983), and latency to LFP response onset of $86 \pm 4 \mathrm{~ms}$ 505 (Prechtl and Bullock, 1994). However, we found that, in responses to a full screen flash 506 or to the change from a blank screen to the start of a complex movie, a typical latency to 507 first spike is around $200-500 \mathrm{~ms}$ which is longer than previously reported latencies. In 508 addition, we provided further support for this range of time delays by looking at the 509 structure of RFs and making it maximally convergent.

510 The effects of adaptation in turtle visual cortex have long been studied. Our 511 investigation stated that the cortex has an adaptation mechanism but did not speak 512 about adaptation in neural pathways leading to the cortex. It is almost certainly the case 513 that both of these effects contribute to the adaptation observed in the cortex. Adaptation 514 in our preparations seems to persist for several seconds to a minute in line with others 515 that showed complete recovery happens in $16 \mathrm{~s}$ (Luo et al., 2010). Though some 516 studies described recovery times in visual cortex ranging from $0.5 \mathrm{~min}$ to 
$5173 \mathrm{~min}$ (Gusel'nikov et al., 1972). We suspect that the apparent discrepancy is due to the 518 different stimuli they used.

Variability we observed in turtle visual cortex has also been seen in cat visual cortex in voltage sensitive dye recordings (Holt et al., 1996; Sadagopan and Ferster, 2012). It

521 has been shown that much of the trial-to-trial variability could actually be explained by

522 the ongoing activity in the cortex (Arieli et al., 1996). That is to say that after subtracting

523 the activity of the cortex immediately preceding the response, the variability of the

524 responses was greatly reduced. More generally, it has been suggested that sensory 525 responses should be thought of as not simply the product of a sensory input and some 526 "default" anatomical connectivity, but instead the product of those along with learned 527 expectations and environmental contingencies that can change continuously (Fontanini 528 and Katz, 2008).

529 A complication our results point to is the presence of a well-defined retinotopic map 530 from the visual field to the cortex. Using retinal ablation and observing orthograde 531 degeneration, Ulinski and Nautiyal (1988) reported that the nasal retina projects to the 532 contralateral rostral lateral geniculate nucleus (LGN). Later, Mulligan and Ulinski (1990) 533 found that the rostral LGN projects to the caudal cortex and the caudal LGN projects to 534 the rostral cortex. Combining these two observations they predicted that the nasal535 temporal axis of visual space is represented along the rostro-caudal axis of the visual 536 cortex. This prediction contradicted earlier results from Mazurskaya(1973), who 537 observed the opposite polarity in recorded evoked potentials in the visual cortex while 538 presenting local visual stimulation to the retina. 
540 the dorso-ventral axis of the LGN, but the data supporting this claim were much less 541 clear than the data supporting conclusions about naso-temporal projections. Continuing

542 along this visual pathway, Mulligan and Ulinski reported that (at least some) neurons in

543 any given dorso-ventral transect of the LGN project along the full lateral-medial extent of

544 the cortex. Thus, a neuron located anywhere along a lateral-medial line in the cortex

545 can respond to stimulation at any point along a particular vertical line in the visual field.

546 With moving dot stimuli, we found that response specificity has variable amplitude but

547 similar patterns for different recording sites. Also, response variations from site to site 548 did not follow any clear trend. We are convinced that experimental data supports our 549 conclusions better than previous studies.

550
What do we learn by comparing visual cortex in turtles with mammals? Inferior temporal cortex (IT) is a visually responsive area in mammals that has a striking resemblance to what we found in turtle cortex. In IT responses to visual stimuli persist up to 15 s (Fuster and Jervey, 1981) and its cells also tend to have large RFs, respond to many stimuli, including moving stimuli, and have adaptation effects with inter-stimulus intervals less than $5 s$ (Gross et al., 1972). Unlike turtle visual cortex strong direction sensitivity has been observed in mammalian IT. Some of the direction sensitive IT cells had one clear preferred direction (termed unidirectional), but most were bidirectional sensitive (they responded preferentially to both a direction and the opposite direction, but not perpendicular motion). This was demonstrated in IT using black bars sweeping across the visual field while we have used black dots that take up only a small portion of the visual field. To scrutinize the similarity, further studies should be done with moving bars that span the entire visual field. 
Mammalian hippocampus and piriform cortex are other areas that have a similar 564 structure to turtle cortex. Like turtle visual cortex, the mammalian hippocampus has 565 extensive feedback connections to its primary input source (entorhinal cortex; Witter, 566 1993). In the hippocampus, like turtle cortex, oscillations are found in the gamma band 567 (Bragin et al., 1995) and theta band with electrodes spanning several hundred microns 568 having similar LFP signals (Buzsáki, 2002).

The olfactory or piriform cortex is a three layer cortical structure, and has 570 feedforward and feedback circuits that are similar to those found in turtle dorsal cortex 571 along with numerous other similarities (Haberly, 1985). Further identifying structural and 572 functional similarities between the turtle dorsal cortex and mammalian piriform cortex 573 will likely help elucidate common organizational and computational principal of cortical 574 networks (Fournier et al., 2014).

Though we have not yet seen the multitude of studies on turtles as we've seen with 576 other preparations such as rat, mouse, and cat; the turtle preparation is becoming more 577 appreciated for allowing the experimenter to study cortical processing from the 578 subcellular level to the level of neuronal ensembles simultaneously, as well as being 579 tolerant enough to a wide range of flexible modifications to meet the needs of a range of 580 experiments with different technical demands.

\section{ACKNOWLEDGEMENTS}

613 We thank members of the Neurophysics Laboratory for helpful discussions. 
615

616

617

618

619

620

621

622

623

624

625

626

627

628

629

630

631

632

633

634

635

636

637

638

639

640

641

642

643

644

645

646

647

\section{CONFLICT OF INTEREST STATEMENT}

No conflict of interest exists for any of the authors.

\section{ROLE OF AUTHORS}

JP and RW devised the study; JP, NW, WC, WS, and RW performed the experiment;

$\mathrm{MH}$ and JP analyzed the data; $\mathrm{MH}, \mathrm{JP}$, and $\mathrm{RW}$ wrote and revised the manuscript.

\section{References}

Arieli A, Sterkin A, Grinvald A, Aertsen A (1996) Dynamics of Ongoing Activity: Explanation of the Large Variability in Evoked Cortical Responses. Science 273:1868-1871.

Bass AH, Andry ML, Glenn Northcutt R (1983) Visual activity in the telencephalon of the painted turtle, Chrysemys picta. Brain Res 263:201-210.

Boiko VP (1980) Responses to visual stimuli in thalamic neurons of the turtle Emys orbicularis. Neurosci Behav Physiol 10:183-188.

Bragin A, Jandó G, Nádasdy Z, Hetke J, Wise K, Buzsáki G (1995) Gamma (40-100 Hz) oscillation in the hippocampus of the behaving rat. J Neurosci 15:47-60.

Butler AB, Hodos W (2005) Comparative Vertebrate Neuroanatomy: Evolution and Adaptation: Second Edition. Hoboken, NJ, USA: John Wiley \& Sons, Inc.

Buzsáki G (2002) Theta oscillations in the hippocampus. Neuron 33:325-340.

Fontanini A, Katz DB (2008) Behavioral states, network states, and sensory response variability. J Neurophysiol 100:1160-1168.

Fournier J, Müller CM, Laurent G (2014) Looking for the roots of cortical sensory computation in three-layered cortices. Curr Opin Neurobiol 31C:119-126.

Fuster J, Jervey J (1981) Inferotemporal neurons distinguish and retain behaviorally relevant features of visual stimuli. Science (80- ) 212.

Gross CG, Rocha-Miranda CE, Bender DB (1972) Visual properties of neurons in inferotemporal cortex of the Macaque. J Neurophysiol 35.

Gusel'nikov V, Pivovarov A (1977) Postsynaptic excitation and in hibition in neurons of the turtle general cortex in response to moving stimuli. Neurophysiology 9:194-199.

Gusel'nikov VI, Morenkov ED, Pivovarov AS (1972) Unit responses of the turtle forebrain to visual stimuli. Neurosci Behav Physiol 5:235-242.

Haberly LB (1985) Neuronal circuitry in olfactory cortex: anatomy and functional implications. Chem Senses 10:219-238. 
648

Holt GR, Softky WR, Koch C, Douglas RJ (1996) Comparison of discharge variability in vitro and in vivo in cat visual cortex neurons. J Neurophysiol 75:1806-1814.

Kajikawa Y, Schroeder CE (2011) How local is the local field potential? Neuron 72:847-858.

Katzner S, Nauhaus I, Benucci A, Bonin V, Ringach DL, Carandini M (2009) Local origin of field potentials in visual cortex. Neuron 61:35-41.

Kriegstein AR (1987) Synaptic responses of cortical pyramidal neurons to light stimulation in the isolated turtle visual system. J Neurosci 7:2488-2492.

Luo Q, Lu H, Lu H, Yang Y, Gao JH (2010) Comparison of visually evoked local field potentials in isolated turtle brain: Patterned versus blank stimulation. J Neurosci Methods 187:26-32.

Mancilla JG, Fowler M, Ulinski PS (1998) Responses of regular spiking and fast spiking cells in turtle visual cortex to light flashes. Vis Neurosci 15:979-993.

Mazurskaya PZ (1973a) Organization of receptive fields in the forebrain of Emys orbicularis. Neurosci Behav Physiol 6:311-318.

Mazurskaya PZ (1973b) Retinal projection in the forebrain of Emys orbicularis. Neurosci Behav Physiol 6:75-82.

Mulligan K a, Ulinski PS (1990) Organization of geniculocortical projections in turtles: isoazimuth lamellae in the visual cortex. J Comp Neurol 296:531-547.

Naumann RK, Ondracek JM, Reiter S, Shein-Idelson M, Tosches MA, Yamawaki TM, Laurent G (2015) The reptilian brain. Curr Biol 25:R317-R321.

Prechtl JC (1994) Visual motion induces synchronous oscillations in turtle visual cortex. Proc Natl Acad Sci U S A 91:12467-12471.

Prechtl JC, Bullock TH (1994) Event-related potentials to omitted visual stimuli in a reptile. Electroencephalogr Clin Neurophysiol 91:54-66.

Prechtl JC, Bullock TH, Kleinfeld D (2000) Direct evidence for local oscillatory current sources and intracortical phase gradients in turtle visual cortex. Proc Natl Acad Sci U S A 97:877882.

Prechtl JC, Cohen LB, Pesaran B, Mitra PP, Kleinfeld D (1997) Visual stimuli induce waves of electrical activity in turtle cortex. Proc Natl Acad Sci U S A 94:7621-7626.

Romer AS, Parsons TS (1977) The Vertebrate Body. 5th ed. Saunders, Philadelphia.

Sadagopan S, Ferster D (2012) Feedforward Origins of Response Variability Underlying Contrast Invariant Orientation Tuning in Cat Visual Cortex. Neuron 74:911-923.

Saha D, Leong K, Katta N, Raman B (2013) Multi-unit recording methods to characterize neural activity in the locust (Schistocerca americana) olfactory circuits. J Vis Exp.

Shew W, Clawson W, Pobst J, Karimipanah Y, Wright NC, Wessel R (2015) Adaptation to sensory input tunes visual cortex to criticality. Nat Phys 11:22-27.

Ulinski PS, Nautiyal J (1988) Organization of retinogeniculate projections in turtles of the generaPseudemys and Chrysemys. J Comp Neurol 276:92-112.

Wiltschko AB, Gage GJ, Berke JD (2008) Wavelet filtering before spike detection preserves waveform shape and enhances single-unit discrimination. J Neurosci Methods 173:34-40.

Witter MP (1993) Organization of the entorhinal-hippocampal system: a review of current anatomical data. Hippocampus 3 Spec No:33-44.

Xing D, Yeh C-I, Shapley R (2009) Spatial spread of the local field potential and its laminar 
690 variation in visual cortex. J Neurosci 29:11540-11549.

691 Ziolo MS, Bertelsen MF (2009) Effects of propofol administered via the supravertebral sinus in 692 red-eared sliders. J Am Vet Med Assoc 234:390-393.

693 
a

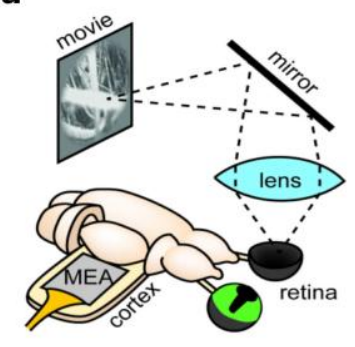

b

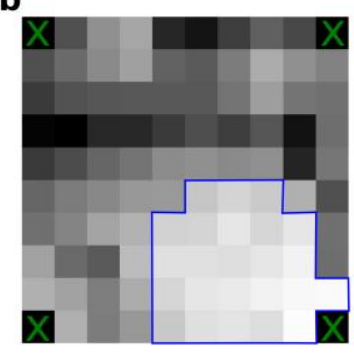

C

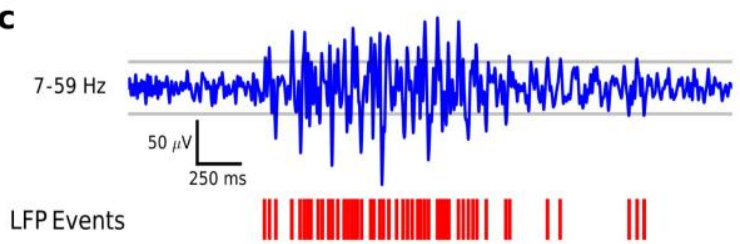

Fig. 1 Experimental setup and quantifying LFP events. a Our experimental set up for experiments done with a monitor and mirror. The visual stimuli are presented on a monitor. The image reflects off a mirror and through a lens to form a picture on the retina of the turtle's hemisected eye. The multielectrode array (MEA) is placed in the unfolded cortex. b Visual responsiveness across the MEA. Each square represents an electrode. The background color for each square indicates strength of their visual responses with black being the weakest and white being the strongest. Enclosed blue region indicates visual responses stronger than the threshold and being included in the analyses. c LFP events are referred to threshold crossings. A filtered LFP response is shown in blue with gray lines as \pm 3 standard deviation, and threshold crossings are marked in red as LFP events. 


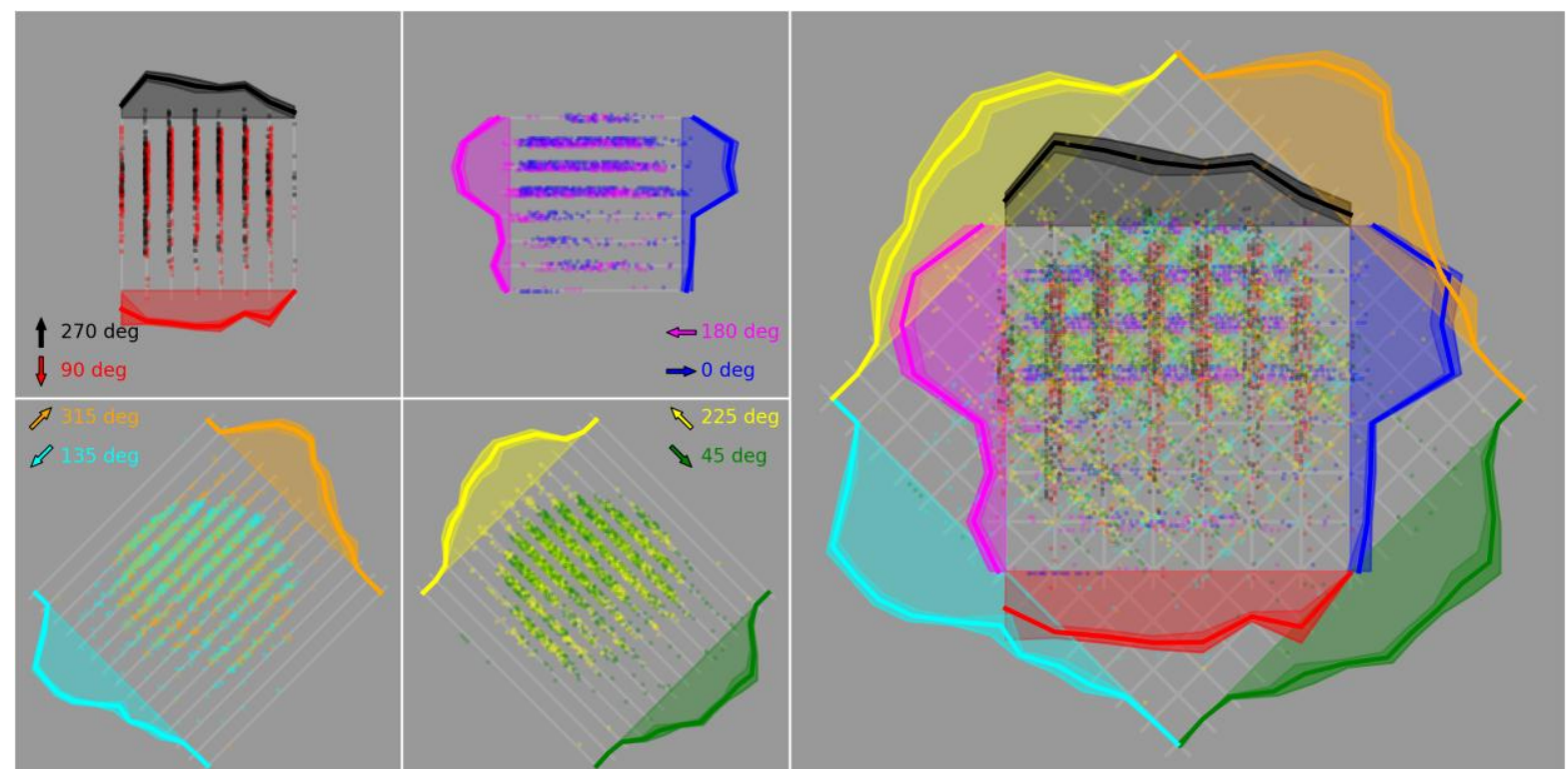

Fig. 2 RF of the LFP signals covers over half of the visual field. Each grey square represents the visual field. Dots move along 8 different paths represented by light grey lines in 8 different directions. LFP events are color coded according to the direction of moving dot. In order to aid with visualization, the LFP events are plotted somewhat offset across each path. The solid lines show the across-trial average of LFP event count with the standard deviation shown as the filled region around the average solid line. On the left we presented the data from one electrode only for dots moving in opposite directions with respect to turtle's visual streak (4 subplots for total of 8 directions). The figure on the right shows all 8 directions together. This shows that this electrode is responding to stimuli presented across a large region of the visual field. Retina is briefly exposed to the dots moving along the first and last path and, therefore, LFP responses are diminished. On the other hand dots presented in the middle paths usually evoke large responses. 

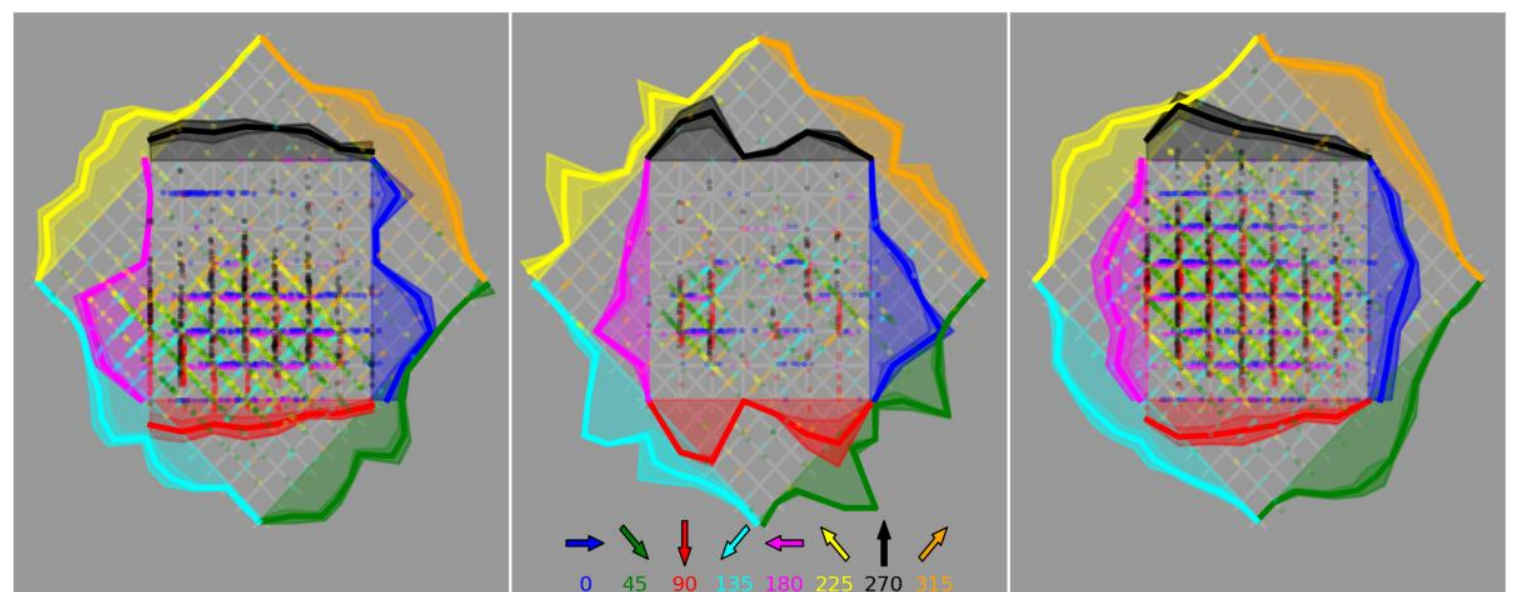

721 Fig. 3 RFs are considerably large for in three different preparations. Responses to black dots moving in different directions on a white screen from the LFP of a single electrode in three different preparations exhibit RFs that cover a considerably large region of the visual field. The middle figure shows that binomial distribution is possible in which middle paths do not evoke strong responses. 


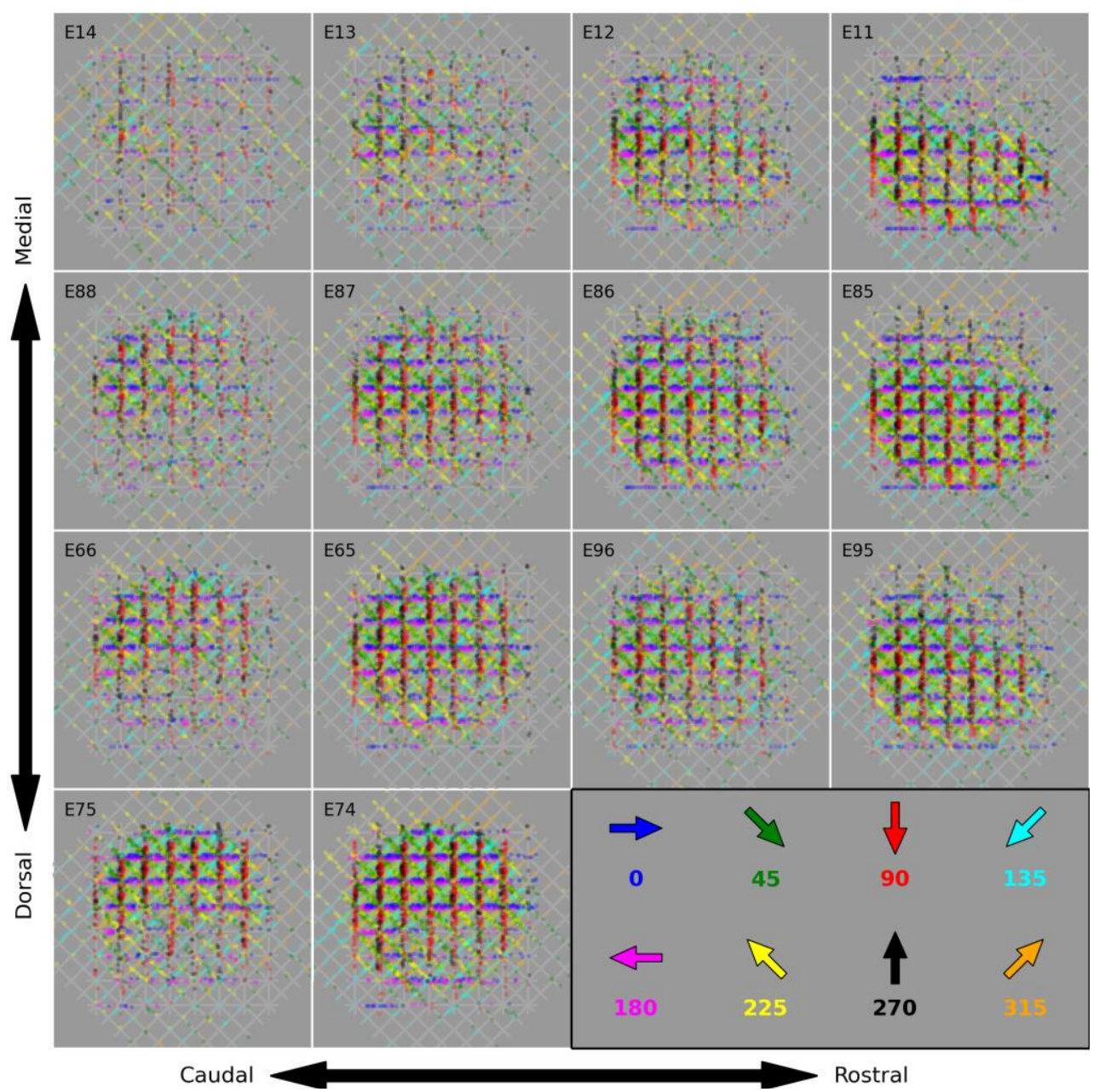

Fig. 4 RF is large across several electrodes. RFs of 14 electrodes in a preparation are shown in response to moving dots. RFs are arranged based on the location of their electrodes in the MEA. This shows that RFs are considerably large for all the responsive electrodes. More importantly, nearby electrodes appear to have more similar RFs than distant pairs (e.g. see E74 and E75 vs. E74 and E85). 

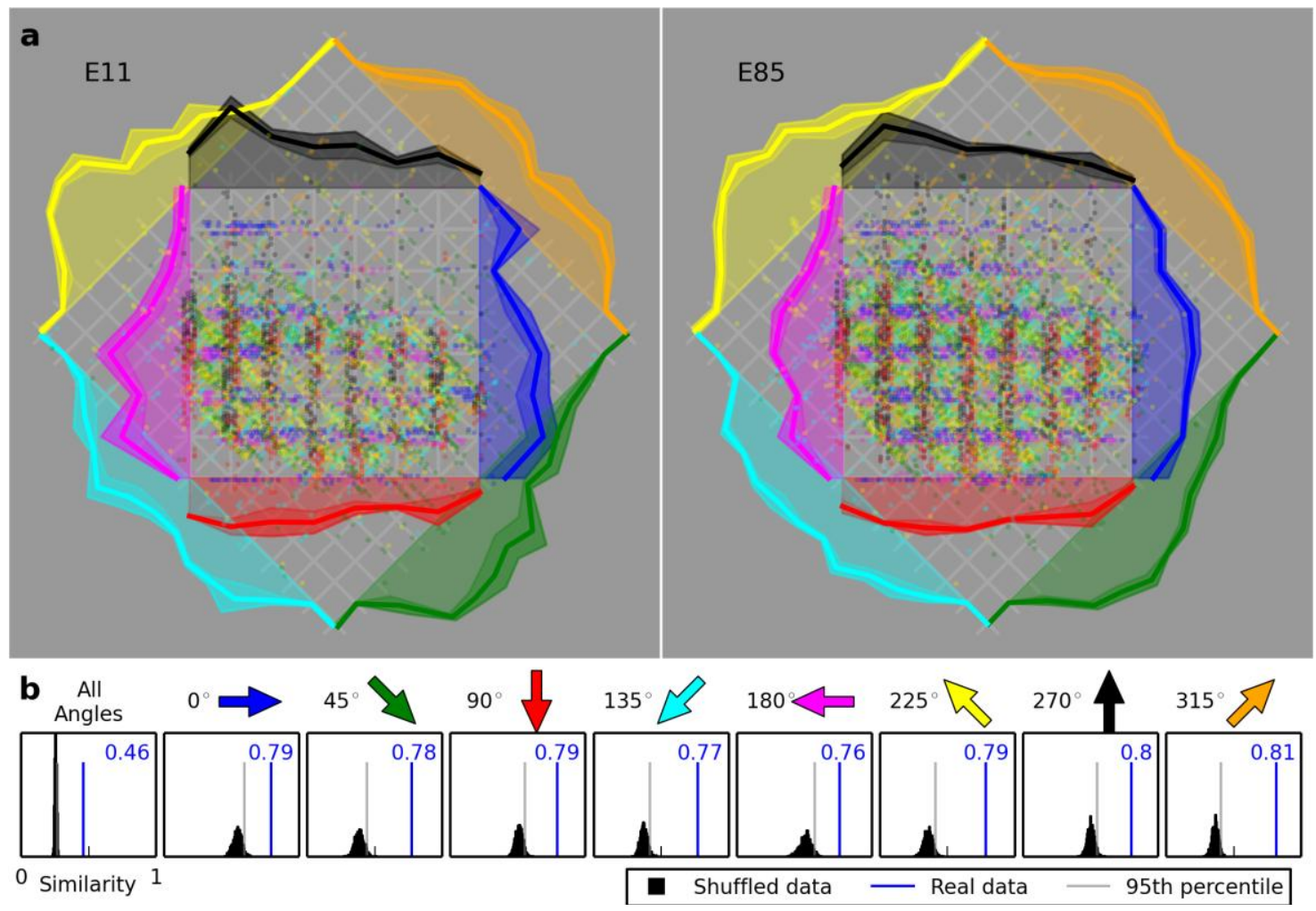

Fig. 5 Nearby electrodes have significantly similar RFs. a Shown are the RFs for a nearby pair of electrodes (E85 and E11; see Fig. 4) as plotted in Fig.2. Note the similarity in the distribution of responses (solid lines) across different directions. $\mathbf{b}$ The similarity between the two RFs when the responses to dots moving at all angles are considered together (the leftmost panel), along with when we consider only the responses to dots moving at a specific angle. The black distribution shows of 1,000 calculated similarities when the data are shuffled. The light grey line shows the similarity below which $95 \%$ of the shuffled similarities lie. The blue line and number show the similarity of the real data for the two electrodes that lie well above the significant level. 


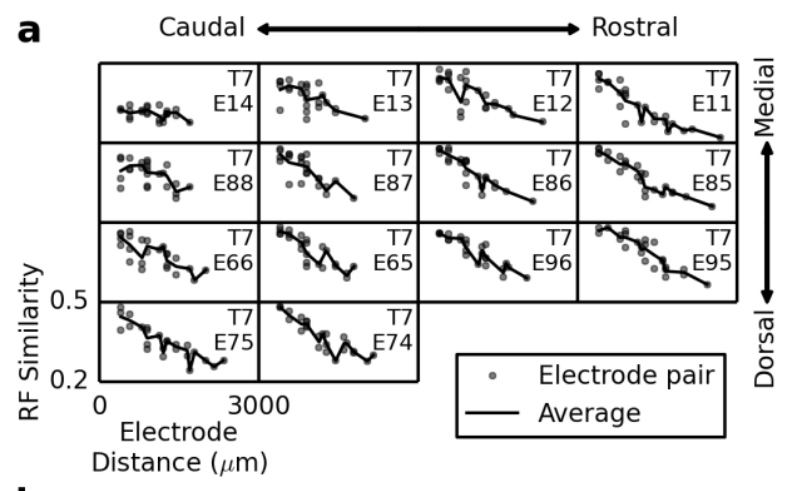

743 Fig. 6 RF similarity gently decays with spatial separation between the electrode pairs. a 744 Plots of RF similarity versus electrode distance. Each plot represents RF similarity of a 745 reference electrode (show in the plot) with all other 13 electrodes (RFs are shown in 746 Fig. 4). Plots are arranged based on the location of their reference electrode in the 747 MEA. In each plot, at a given distance, each point is the similarity of the RF of the 748 reference electrode with another visually responsive electrode separated with that distance. Solid line shows the average RF similarity. b The average RF similarity at each electrode pair distance for all visually responsive electrode pairs for four turtles. *Turtle 5 was included here to show that its trend is consistent with the others, but the visual responses for turtle 5 were relatively weak. Therefore, in order to have enough visually responsive electrode pairs for turtle 5 we used a lower threshold $(0.5)$ for visual 754 responsiveness. 


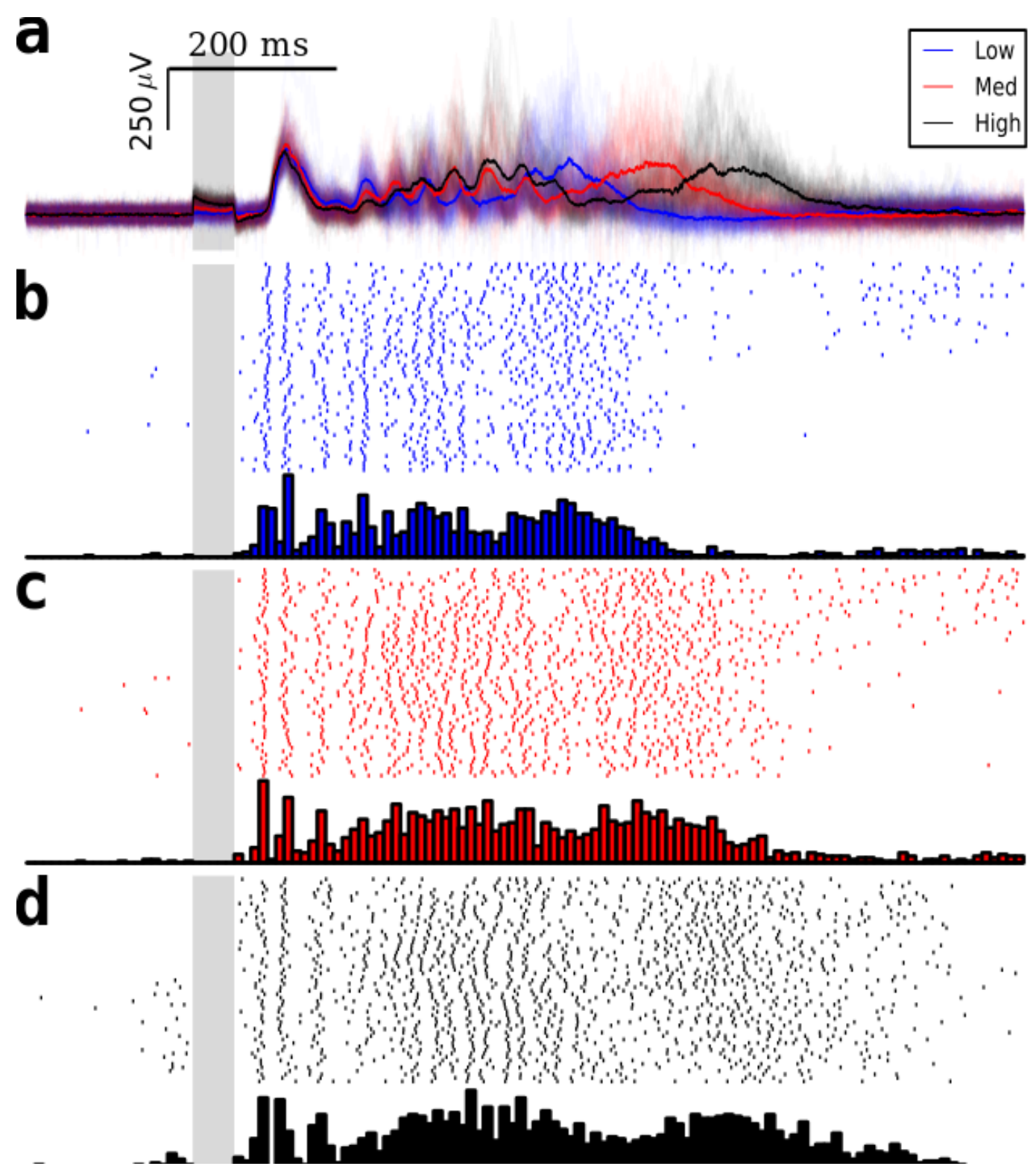

Fig. 7 Temporal structure of the responses evoked by brief LED flashes. a Individual LFP responses in low opacity and average responses in bold for three amplitudes (blue, red, and black for low, medium, and high amplitudes respectively) of $50 \mathrm{~ms}$ LED flashes with $30 \mathrm{~s}$ inter-trial intervals. Early LFP responses consist of several hundred millisecond oscillations dominated by one or two frequencies while later responses cover a broad range of frequency spectrum. b Rastergram of LFP events (top) with prestimulus time histogram (PSTH; bottom) in response to low-amplitude flashes. Rastergram reveals the presence of oscillations in early responses. $\mathbf{C}$ and $\mathbf{d}$ Same as in b for medium and high flash amplitudes. 


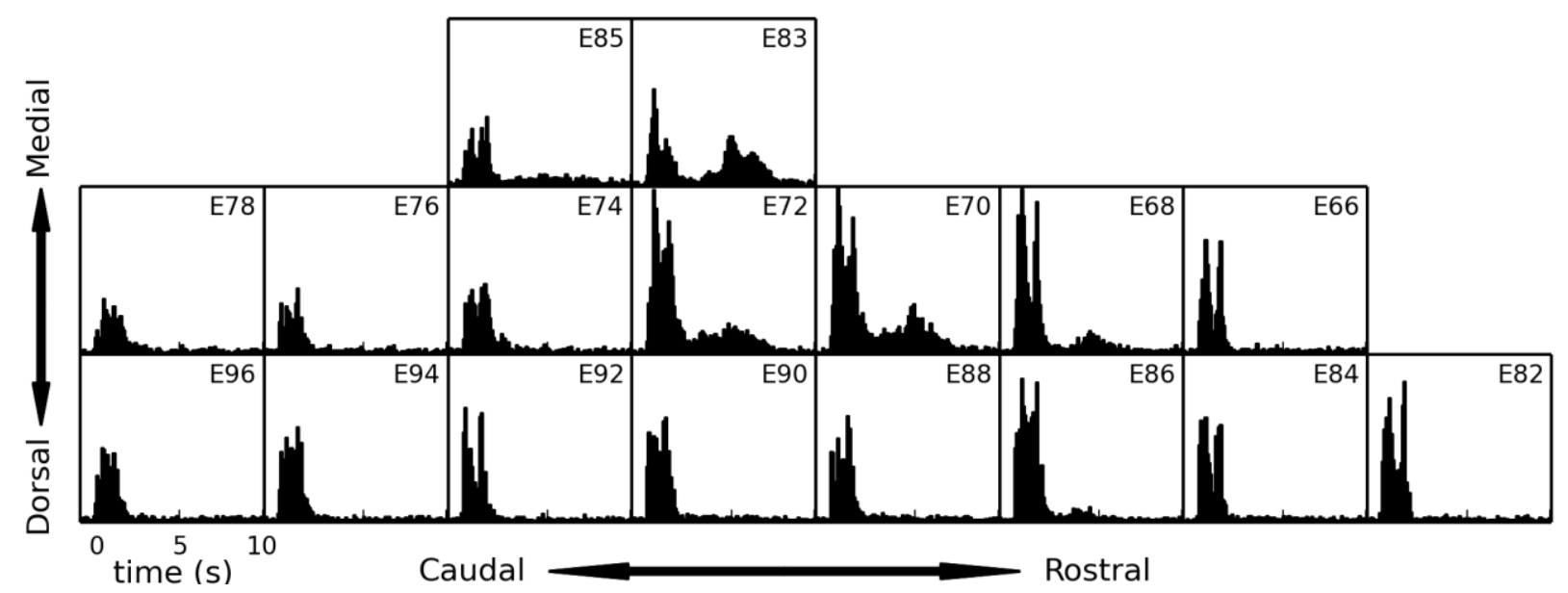

Fig. 8 Persistent response happens on several electrodes. PSTHs are calculated over an extended period of time (10 s window) for several electrodes in response to a brief $50 \mathrm{~ms}$ LED flash. Plots are arranged based on the location of their corresponding electrodes in MEA. A second wave of activity occurs several seconds after stimulus onset and lasts for several seconds. 


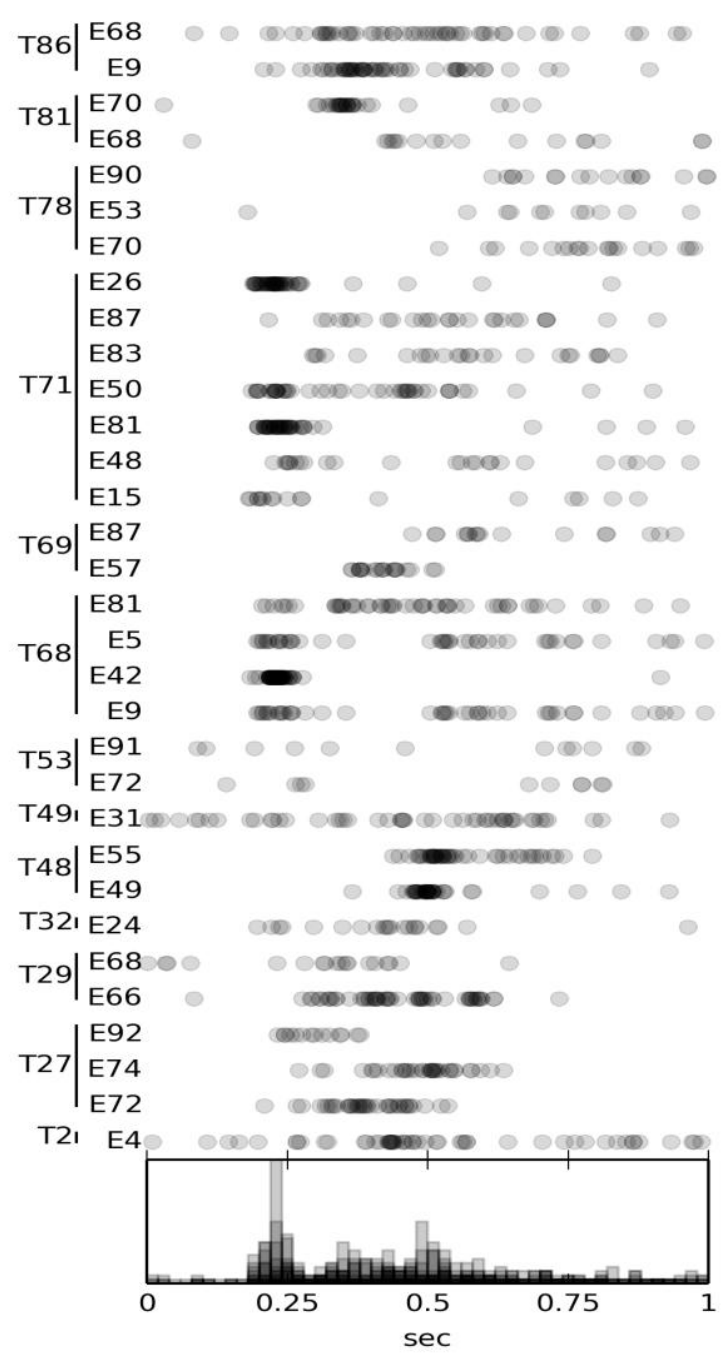

Fig. 9 A typical latency to first evoked spike is around $200-500 \mathrm{~ms}$. The time of the first detected spikes following either the onset of a red LED flash or the transition from a blank screen to the beginning of a complex movie are marked by grey circles. Latencies are shown for several trials of 32 channels across 13 different turtles. Data exhibits a significantly wide range for latency among channels, trials, and turtles. Summary histogram is shown at the bottom which shows a typical 200-500 ms delay. Same wavelet filtering technique is used to detect spikes, but with different parameters (Daubechies wavelets with minimum level of 3 , maximum level of 7 , minimum frequency of $117 \mathrm{~Hz}$, and maximum frequency of $3750 \mathrm{~Hz}$ with a 10 times standard deviation as threshold). 


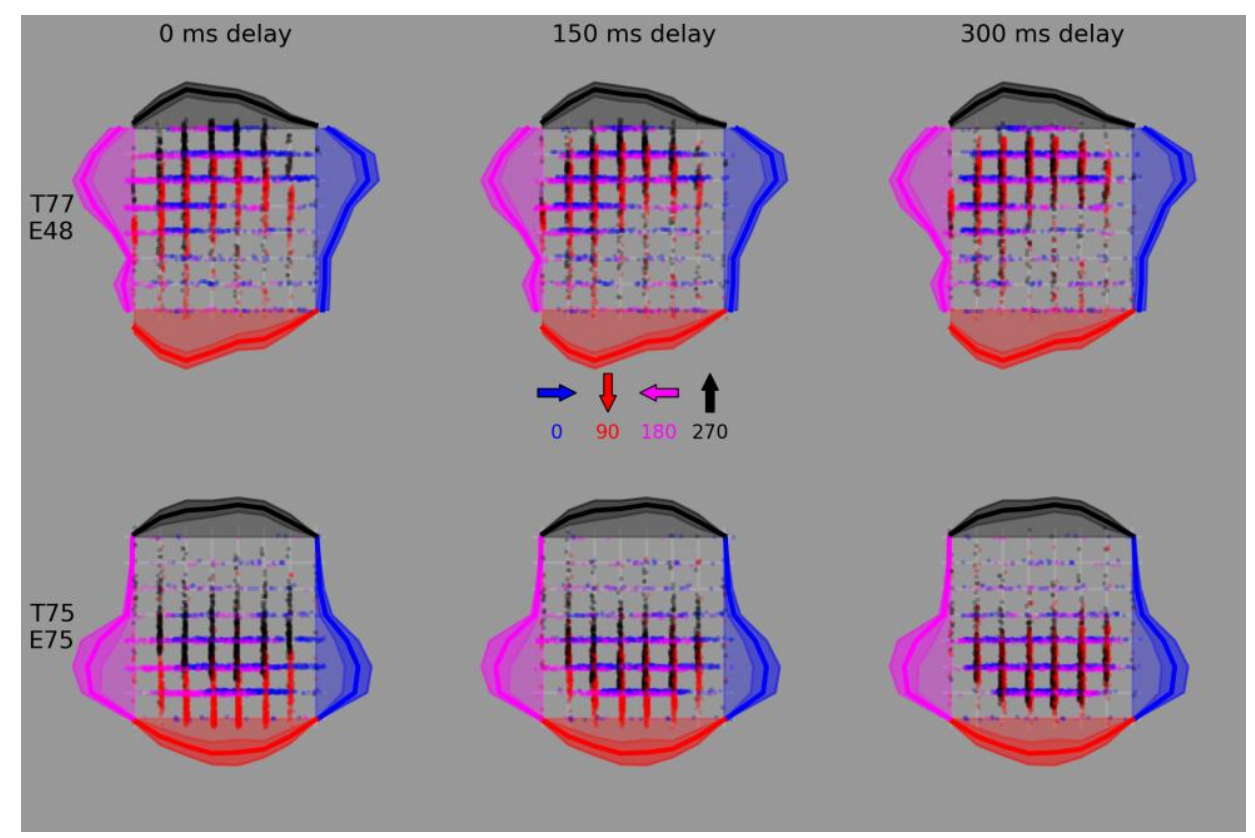

791 Fig. 10 RFs converge most with delays of $\sim 300 \mathrm{~ms}$. Applying a range of delays $792(0,150,300 \mathrm{~ms})$ for two electrodes of two different turtles across several moving dot 793 directions. Detected LFP events are attributed to the region of visual field in which the 794 dot was at prior time. We see the most overlap of the contributions to the visual field 795 from dots moving at different angles for a $300 \mathrm{~ms}$ time delay. 
a

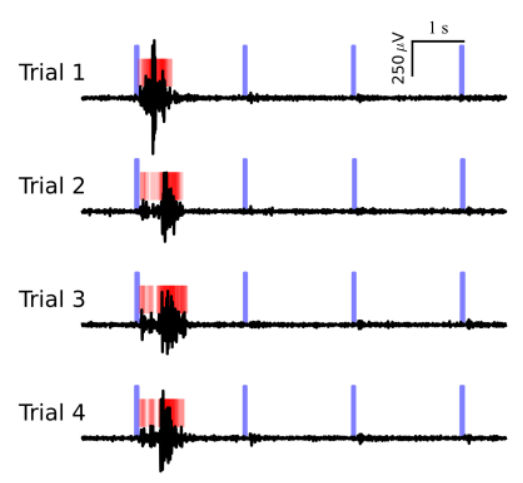

C

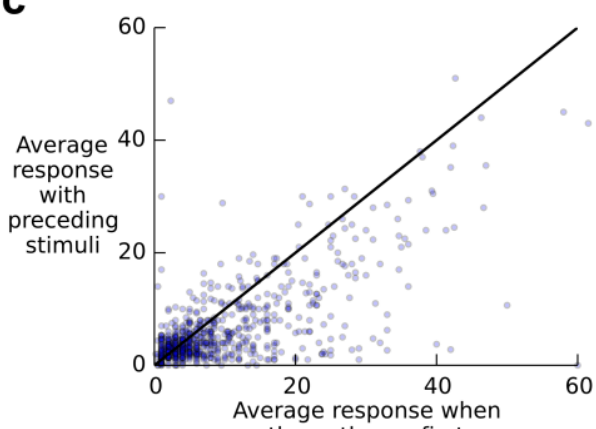

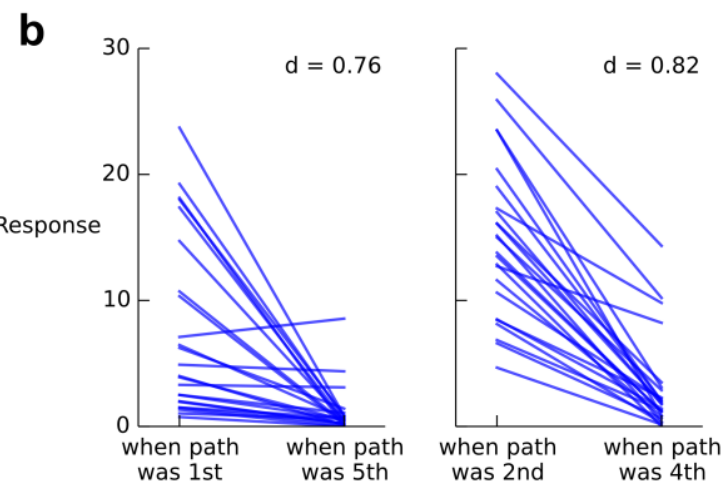

d

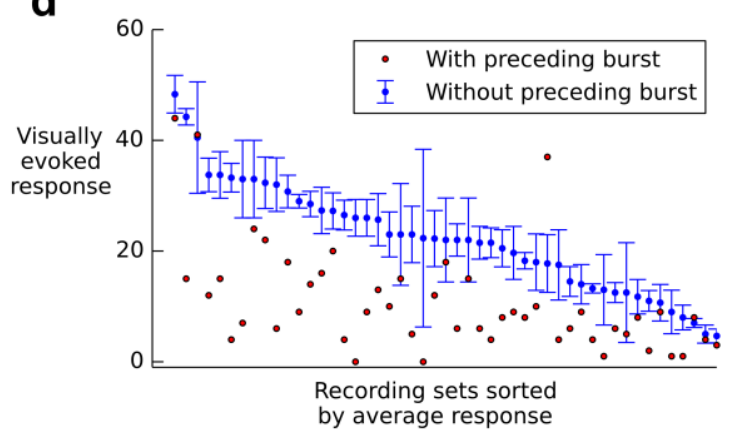

Fig. 11 Cortical adaptation to evoked and ongoing activity. a LFP responses to four repeated presentations of a series of four brief full field flashes (flash timing indicated by blue vertical bars; $100 \mathrm{~ms}$ flashes with $2 s$ inter-flash-interval). LFP threshold crossings are indicated by red rasters. Adaptation abolishes responses to subsequent flashes. b The responses to dots moving along 5 paths across visual field greatly adapt. For each path the response strengths when the path was an early stimulus in the series of stimuli (i.e., either the first or second path to be presented) is compared against the response strengths when the same path was presented later in the series of stimuli (i.e., either the fourth or fifth path). Each point plotted is the average of 7-30 trials. The average decrease in response strength, $d$, is 0.76 (left) and 0.82 (right). c Average response with preceding stimuli is significantly smaller that first path average response $(p<<$ 0.001). d Response strengths with and without preceding spontaneous activity. We show visual responses from several different stimuli. Red dots indicate individual responses to visual stimuli that were preceded by a strong burst of spontaneous activity within a $5 s$ window before the stimulus. For each of those recordings, the average response of the 2-4 trials of the same stimulus nearest in time to the recording that was preceded by a burst is shown in blue with error bars showing the standard deviation. The 2-4 reference recordings were selected from recordings that were not preceded by a spontaneous burst of activity. Each recording preceded by a spontaneous burst together with the 2-4 reference recordings are collectively referred to as a recording set. Clearly spontaneous activity leads to a significant and reliable adaptation of subsequent visual responses. 


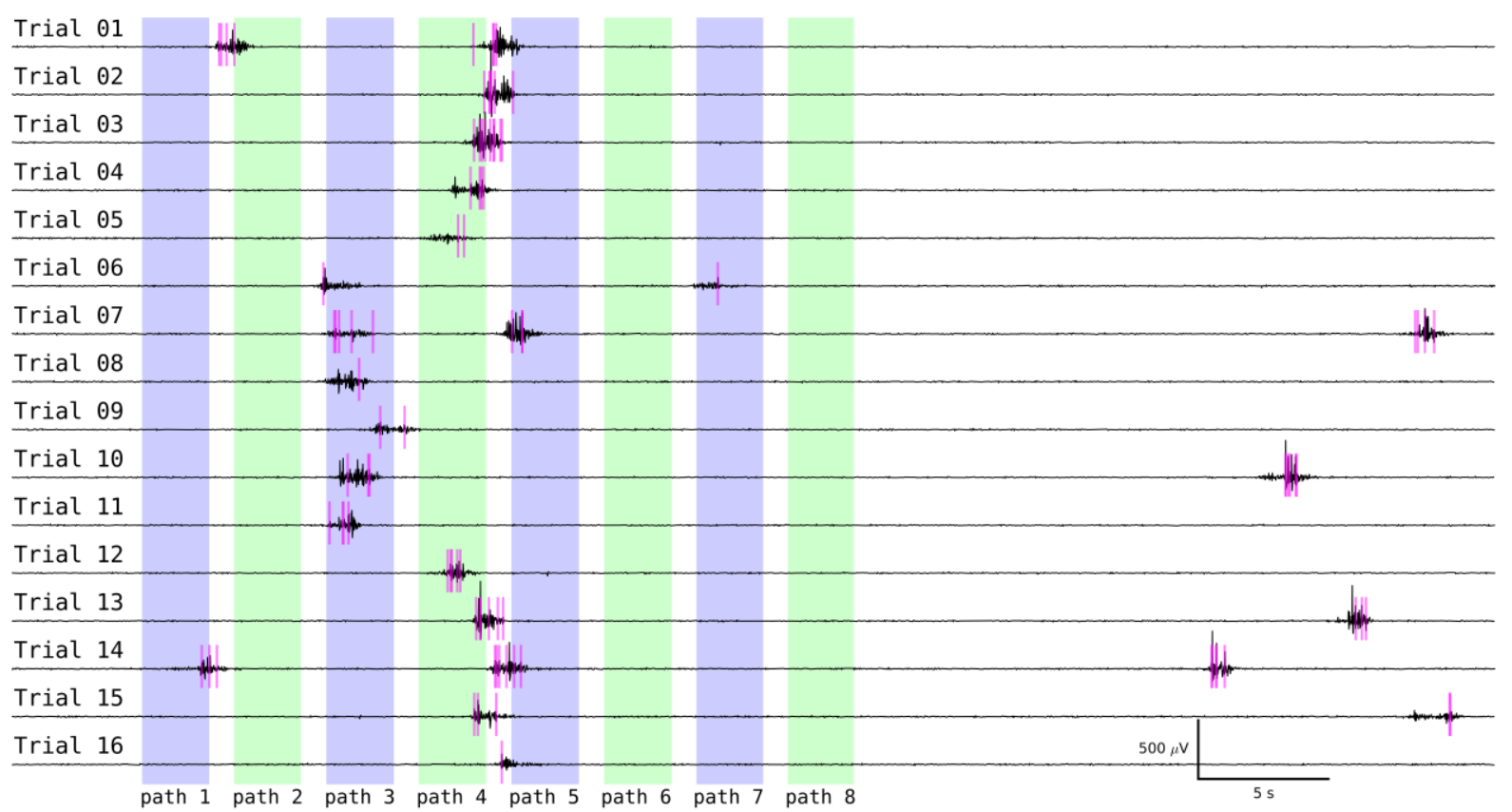

Fig. 12 Responses exhibit variable strength, onset timing, or temporal properties. LFP signal (black) with action potentials (magenta rasters) during 16 trials of 8 dots moving along 8 different paths in one direction. The 8 colored columns indicate the timing of the 8 dots moving across the visual field. If we compare the responses to the $3^{\text {rd }}$ path in the $7^{\text {th }}$ and $8^{\text {th }}$ trial, we find markedly different response amplitudes. Response onset time to the $4^{\text {th }}$ path shows variability of order of several seconds as well as in how that response plays out. For some trials (e.g., trials 1-3) we see roughly one large burst, and for others 827 (e.g., trial 4) it looks more like a series of two smaller bursts. 

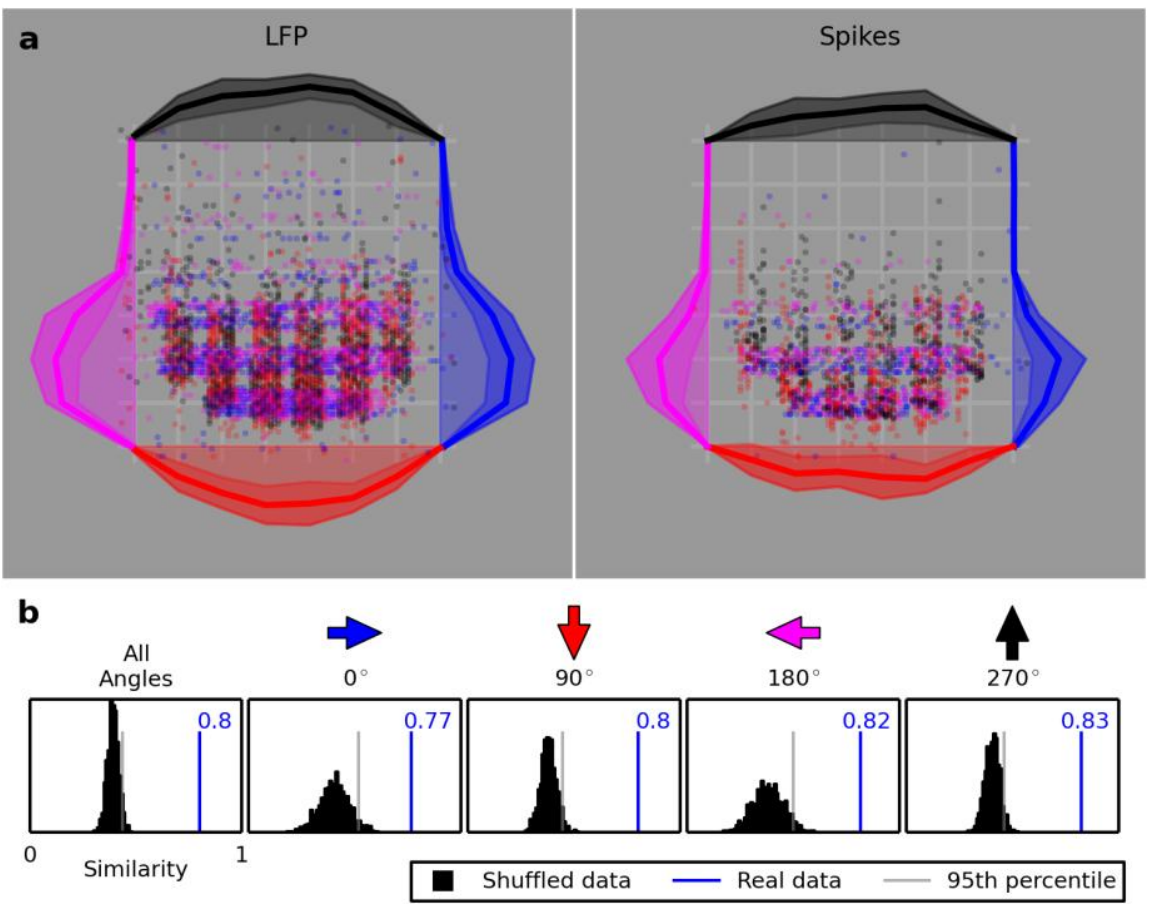

829 Fig. 13 Spike-LFP RF exhibits a significant similarity. a The LFP (left) and spike (right) RFs for one electrode are plotted. The spike RF is obviously smaller that the LFP RF but still considerably large. $\mathbf{b}$ The similarity between the two RFs when the responses to dots moving at all angles are considered together, along with the similarity separately for different angles. The black distribution shows of 1,000 calculated similarities when the data are shuffled. The blue line and number show the similarity of the real data, and the light grey line shows the similarity below which $95 \%$ of the shuffled similarities lie. 
a
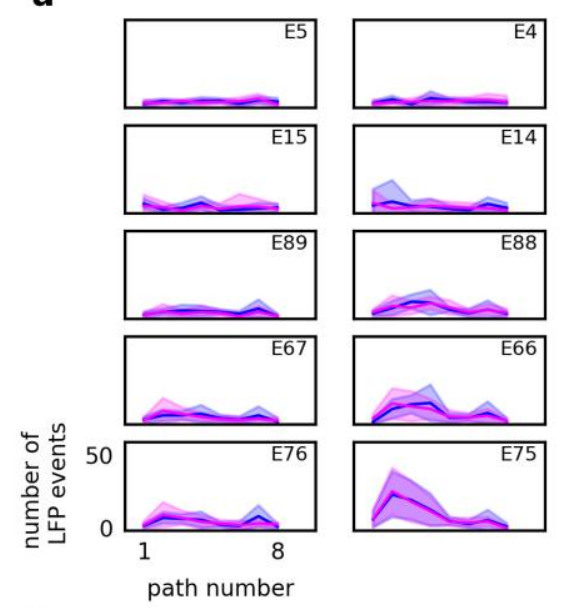

b
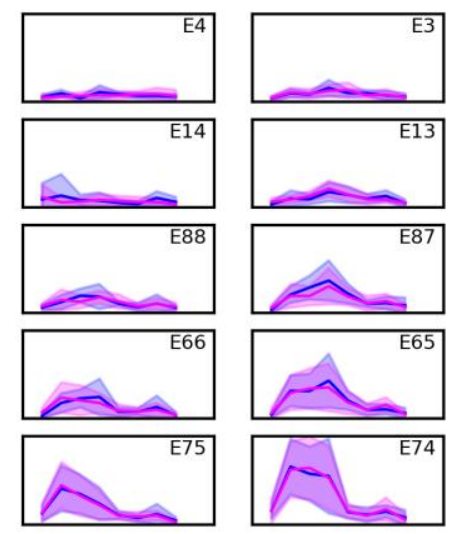

path number
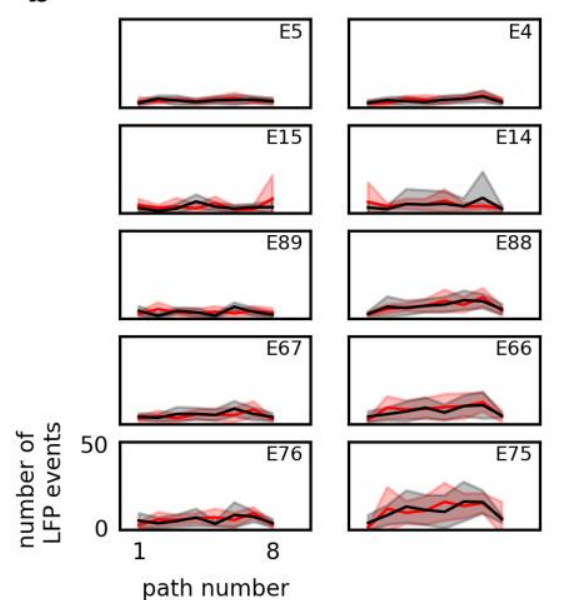
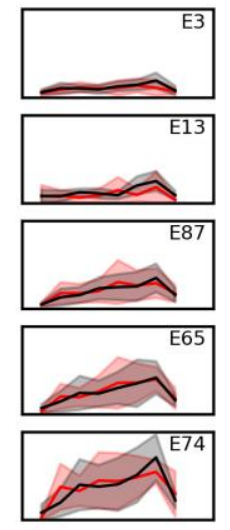
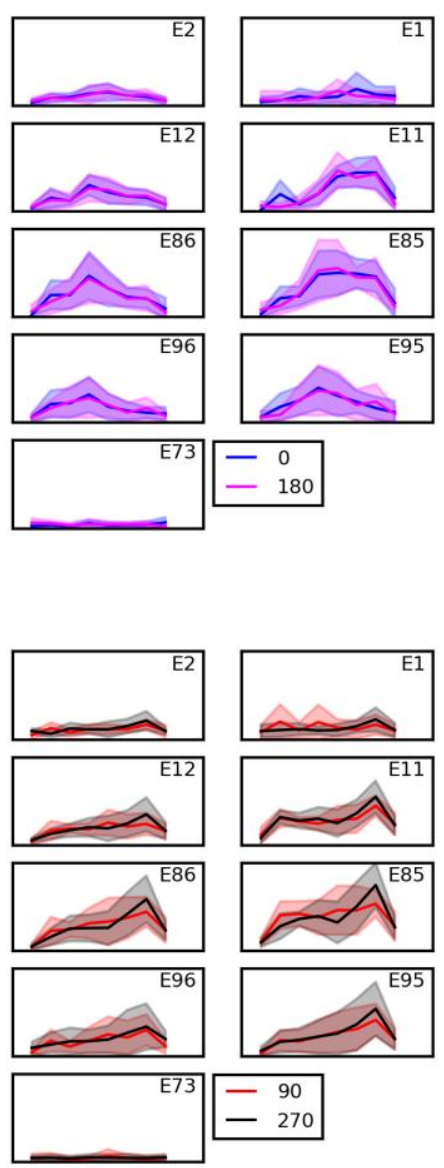<smiles>C1=C[C@H]2CC[C@H]1C2</smiles>

836

837

838

839

840

841

842

843

844

845

846

Fig. 14 No clear mapping from the visual field to the visual cortex exist. a Nasotemporal response specificity has variable amplitude but similar patterns for the recording sites on MEA. For 24 electrodes we show the average responses (lines) and standard deviations (filled area around the lines), in response to dots moving along 8 different vertical paths arranged naso-temporally in the visual field. The two colors represent the two opposite angles that traverse the paths. Response strength varies but not the pattern and, moreover, the variations from site to site did not follow any clear trend. b LFP responses to dots moving along 8 paths arranged vertically in the visual field plotted in the same format as in a. The two colors represent the two opposite angles. 